\title{
Optimization of Meridional Flow Channel Design of Pump Impeller
}

\author{
Sunao Miyauchi* \\ KUBOTA Corporation, Pumps R\&D Department, 1-1-1 Hama, Amagasaki-City, 661-8567, Japan
}

Hironori Horiguchi and Jun-ichirou Fukutomi

Department of Mech. Engineering, The University of Tokushima, 2-1 Minami-Josanjima, Tokushima-City, 770-8506, Japan

\author{
Akihiro Takahashi \\ KUBOTA Corporation, Pumps R\&D Department, 1-1-1 Hama, Amagasaki-City, 661-8567, Japan
}

The meridional flow channel design of a pump impeller affects its performance. However, since so many design parameters exist, a new design method is proposed in which a meridional and blade-to-blade flow channel is designed by the parallel use of the circulation distribution provided by the designer. Thus, an optimization method was used to design an axis-symmetrical meridional flow channel from the circulation distribution. In addition, the inverse design method proposed by Zangeneh et al. (1996) was employed to design a three-dimensional blade-to-blade flow channel from the circulation distribution and the optimized meridional shape. In this article, a few design examples and these Computational Fluid Dynamics (CFD) validations are also given.

Keywords Pump, Meridional flow channel, Optimization, Inverse design, New design method

\section{OUTLINE OF ISSUES AND MOVEMENTS ON PUMP IMPELLER DESIGN METHOD AS SEEN BY A DESIGNER}

The inside of an impeller in turbo machinery such as pumps is a flow field in which extremely complex three-dimensional flows occur with the development and separation of boundary layers, various vortexes, and the occurrence of secondary flow. Therefore, even when an analysis is conducted, results, which differ greatly from the actual phenomenon, are observed. Information itself becomes a large noise with a small signal to noise

Received 25 June 2003; in final form 10 September 2003.

${ }^{*}$ Corresponding author. Tel.: 81-6-6470-5777, Fax: 81-6-64705800. E-mail: sunao-m@kubota.co.jp ratio and it is difficult to grasp the essential mechanism to improve performance. Also, by the mesh dependency of vortexes and shear flows with a large velocity gradient, and moreover, since physical models such as non-closed and currently branching turbulence models generate various levels of noise (analysis error), the analysis results will depend greatly upon their objects and processes. Consequently, the study of the appropriateness of the mesh or the turbulence model often becomes more central than the phenomenon itself. Therefore, designers tend to think that it is more accurate and expeditious to make an actual prototype impeller and take the actual measurements by Rapid Prototyping-like Laser Stereo-lithography. On the other hand, in the design of impellers as in the past, design parameters, which are deeply related to performance, are clarified first. These are in regard to the meridional flow channel and blade-to-blade flow channel which are flow fields relatively independent of each other but which greatly affect performance. Thus, their effectivity and flow mechanisms were studied (Senoo and Nakase, 1972; Dallenbach, 1961). For example, Mishina theorized in detail on the relation between performance and relative velocity distribution and circulation distribution (Mishina, 1978).

Recently, in regard to blade-to-blade flow channel, Zangeneh et al. (1996) made a practical three-dimensional inverse design method which can be effectively used to develop actual turbo machines. When circulation distribution $(R C u)$ and meridional shape (which are the design parameters that relate directly to performance) are given, an impeller shape with the desired performance may be obtained and therefore, the method is selfcompleting, clear, and a satisfactory design method with good perspective. Next, a flow analysis is made incidentally on the design results by the Navier-Stokes Equation (N-S) method. Since the object of analysis is appropriate, convergence quality is good and therefore, the effect by boundary layers and secondary flow 
on performance such as efficiency can be appropriately and sufficiently verified. The method is also rational from the point of research, development, and productivity in man-hours. Dallenbach (1961) very appropriately emphasized the importance of the design method when he said, "The narrowing down of the degree of freedom of design within an unlimited number of impeller shapes with a large degree of freedom and making a nearly optimum impeller is the object of the design method." Also, on the effectiveness of the inverse design method based on the flow field, Zangeneh, Goto, and Takemura said, "The flow field gives insight to experienced technicians." It can be said that his insight, gained from accumulated experience in producing many designs and the actual flow phenomenon, systemized in a design concept form, is fitting of a designer of his experience and stature and that further strength may be gained by contact with his design method based on flow fields. In addition, even with simple shaped defusers, the flow is greatly changed and separation or reverse flow occurs by a slight changing of the inclination angle.

The effect given by the thickness of the inlet boundary layers is great and the flow phenomenon is greatly dependent on the flow itself with an unlimited degree of freedom rather than on the flow channel shape. Furthermore, the flow phenomenon is not only complex but consists of layer characteristics like the main flow, various shear flows arising secondarily from the main flow, and minute turbulence structures. Since the handling of physical models of lower layers becomes more complex, it is important to determine the layer at which the technical pursuit of the impeller design should be discontinued. The present inverse analysis design method can be said to be a method which embodies the perspectives and design sense of flow fields, including those above, in actual methods. On the other hand, the meridional flow channel also greatly affects pump performance resulting in events such as cavitation but there are too many flow design parameters which must be satisfied. Also, since there are many structural and functional demands of the various levels from the actual pump field, designs which satisfy the many design parameters were made while making trade-offs through trial and error. Therefore, it would seem that the latest optimization de-

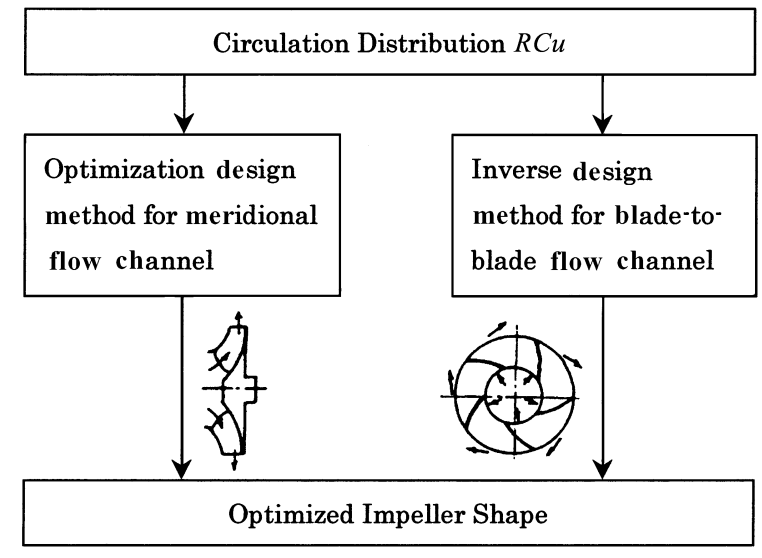

FIGURE 1

Flow of new design method.

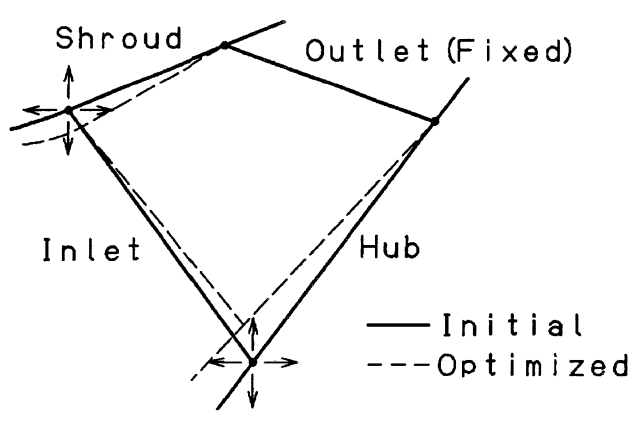

FIGURE 2

Initial shape with inlet design parameters.

sign method, which pursues the Pareto solution of multipurpose design problems, may be used.

Consequently, a new design method is proposed in Figure 1 where the designer gives the circulation distribution $(R C u)$ and the meridional flow channel and blade-to-blade flow channel are designed in parallel. The latest optimization design method is used for the meridional flow channel while the inverse design method is used for the blade-to-blade flow channel.

\section{OUTLINE AND RESULTS OF THE NEW DESIGN METHOD, MAINLY WITH THE MERIDIONAL FLOW CHANNEL OPTIMIZATION}

The application of optimization to the flow field was conducted relatively early on but only recently has it become a practical design tool since the analysis load is large and the flow design parameters of the meridional flow channel are numerous (Vanderplaats and Hicks, 1976).

The present case introduces an example where the design was created by the integrated design optimization software, VisualDOC, and the flow analysis software, FLUENT, using a

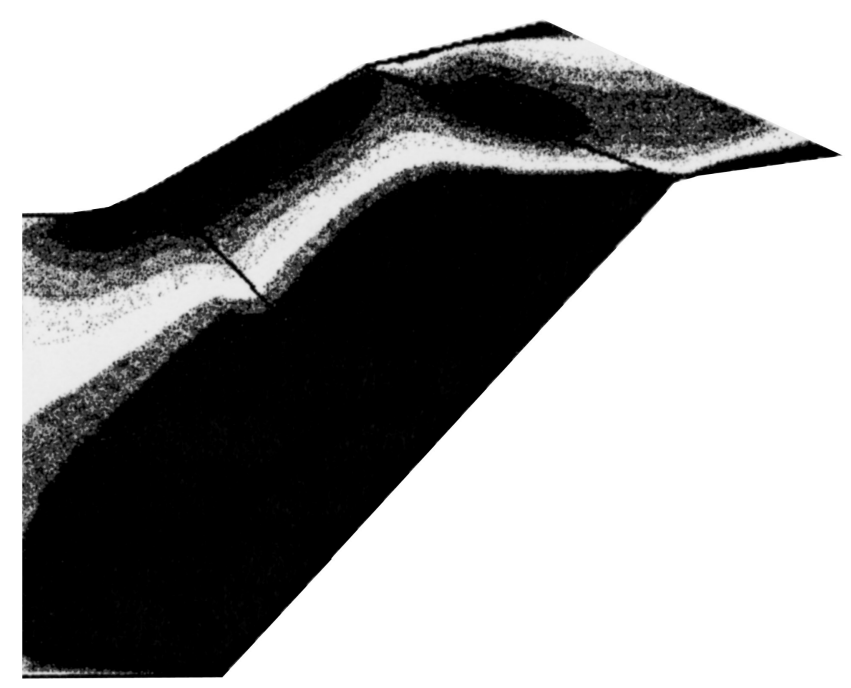

FIGURE 3

Optimized shape with $\mathrm{Cm}$ contour. 


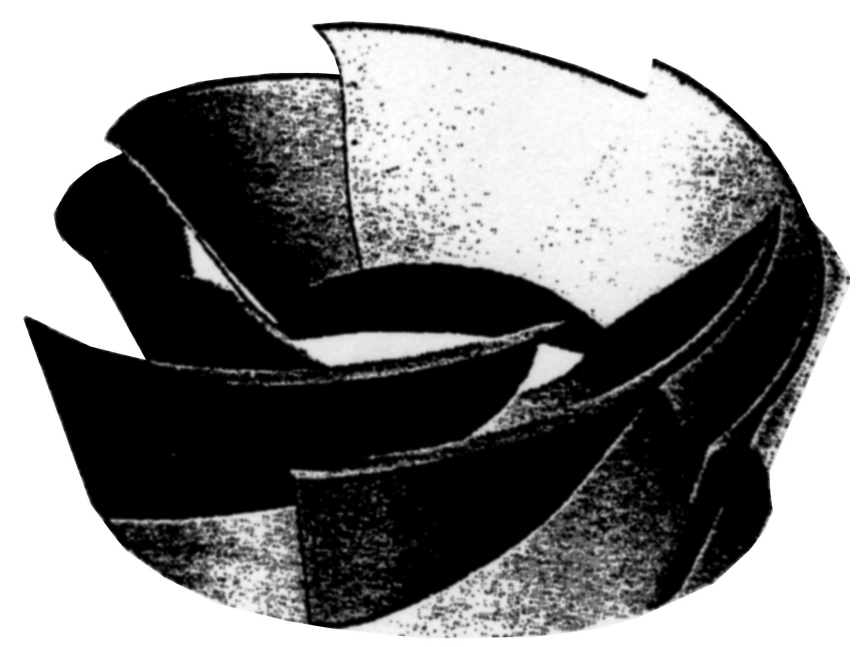

FIGURE 4

Optimized impeller shape.

relatively small number of variables in regard to a mixed flow impeller.

1. How to obtain optimization.

From a numerical experiment by an orthogonal chart (4 variables, 3 levels), the response surface (secondary polynomial) is obtained; a search is made by MMFD and optimized.

(Advantages of the response surface methodology)

- Upon obtaining a multiple number of response surfaces, it becomes possible to study multipurpose, multistage optimization by combining the obtained response surfaces.
- Since non-linear problems can be handled algebraically, the efficiency and robustness of optimization is high. Also, it is strongly resistant to analytical error noise.

2. Analysis method on flow of the meridional flow channel.

- To be a two-dimensional, axis-symmetrical annular shape flow channel without blades.

- As a model of blades, the total pressure rise (theoretical pump head Hth) equivalent to circulation $R C u$ is given, almost linear from the blade inlet to outlet.

- Swirl velocity $C u=g$. Hth/U.

3. Initial shape.

A mixed-flow pump with a specific speed of $N s=900$ $\left(\mathrm{m}^{3} / \mathrm{min}^{\mathrm{m}} \mathrm{m}, \mathrm{min}^{-1}\right)$ as shown in Figure 2. The broken line indicates the optimized shape.

4. Design variables.

Coordinates of the hub and shroud of the blade inlet (Figure 2)

5. Objective function.

The present decrease ratio of average relative velocity at the shroud $W_{2} / W_{1}=0.91$.

6. Optimized shape and its meridional velocity $\mathrm{Cm}$ distribution.

As shown in Figure 3, the somewhat slow part of $\mathrm{Cm}$ seen on the hub side is thought to be caused by the total pressure rise and centrifugal force by swirl. However, an almost even $\mathrm{Cm}$ distribution is observed at the outlet.

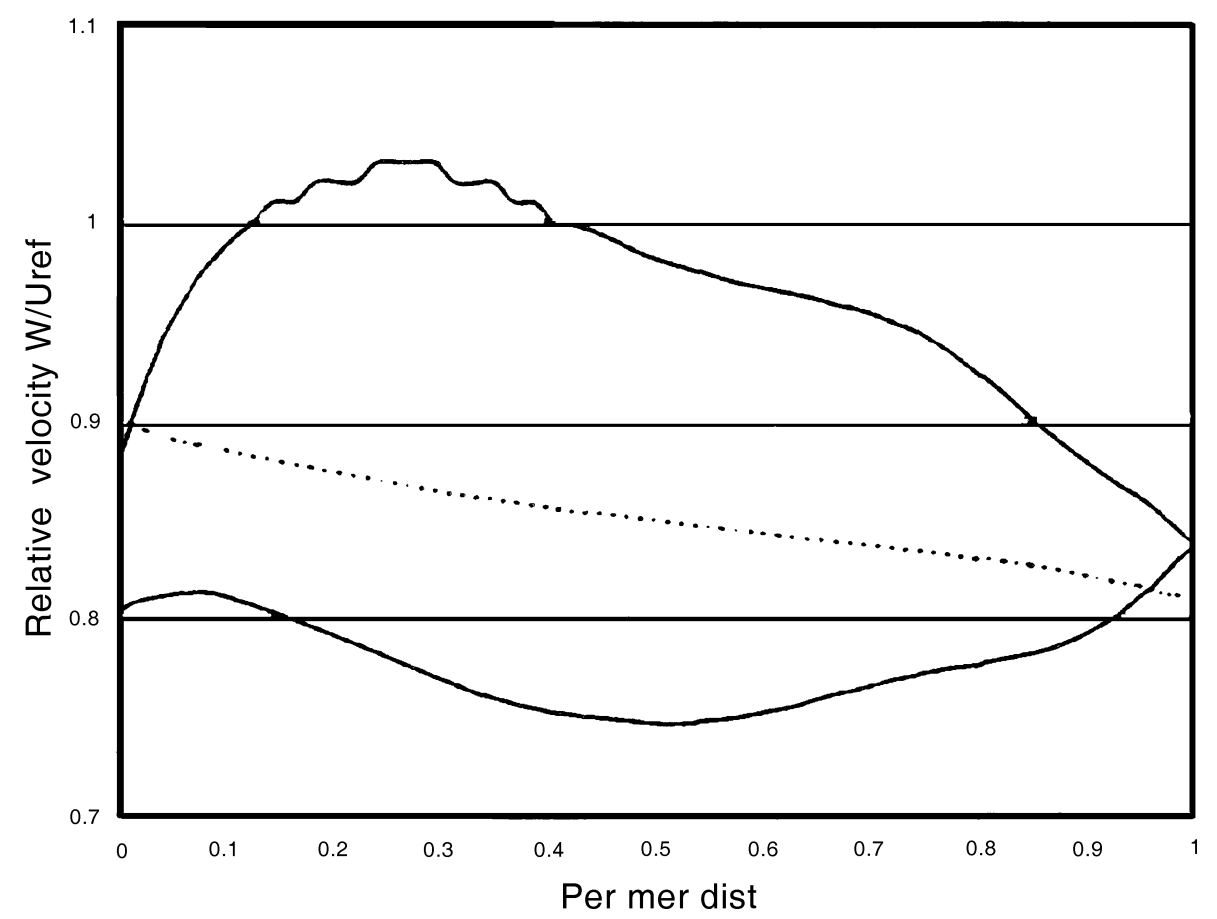

FIGURE 5

Relative velocity distribution of the shroud suction and pressure surface. 


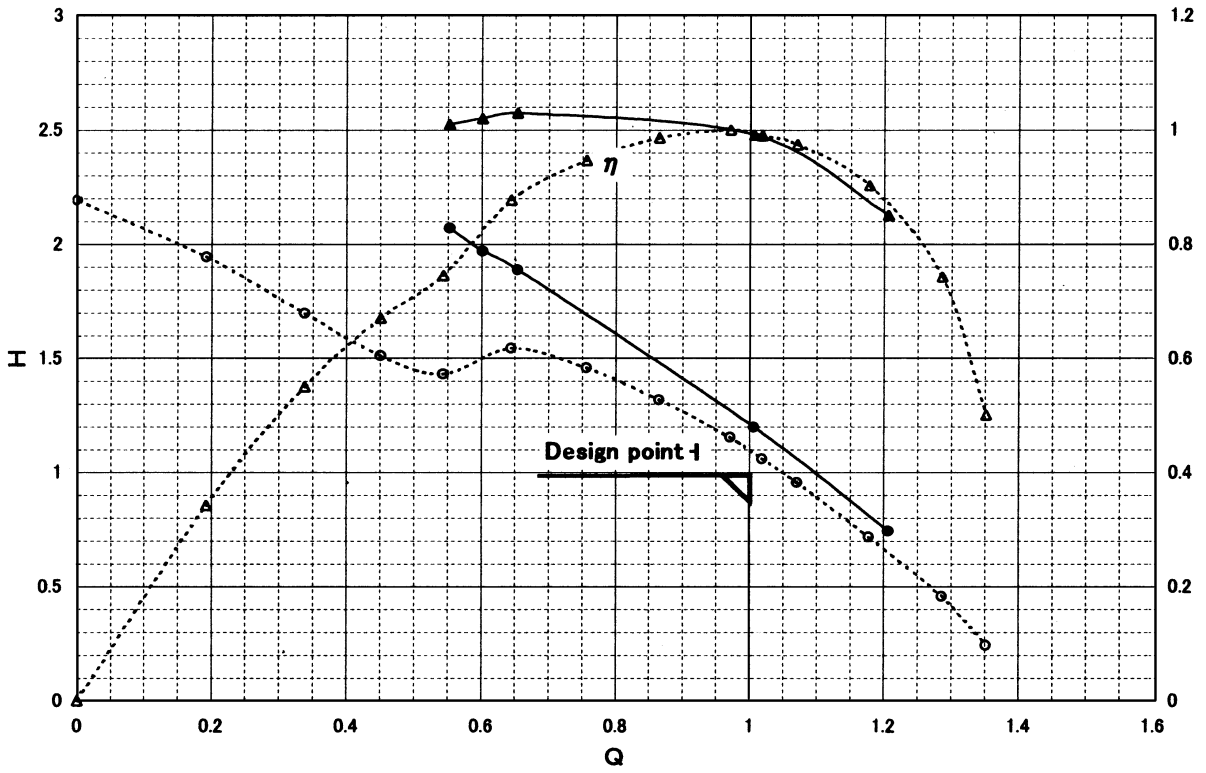

FIGURE 6

Performance curve by analysis of the optimized impeller shape.

7. Inverse design of blade-to-blade flow channel.

The impeller shape shown in Figure 4 was designed by the three-dimensional inverse design method software, TURBOdesign, using the present meridional flow channel and the above-mentioned circulation distribution $R C u$.

In this method, the meridional derivative of the circulation, which is related to blade loading, is controlled by the 4-blade loading design parameters at the hub and shroud, which are carefully given by the designer. Next, vortex sheet strength representing the blade cascade is determined by this distribution and the blade shape is determined so as to be aligned with the local velocity induced by the vortex sheet.

Figure 5 shows the relative velocity distribution of the shroud suction and pressure surface, where the average relative velocity $W$ (broken line), previously obtained by the meridional flow channel optimization, lies approximately midway between them.

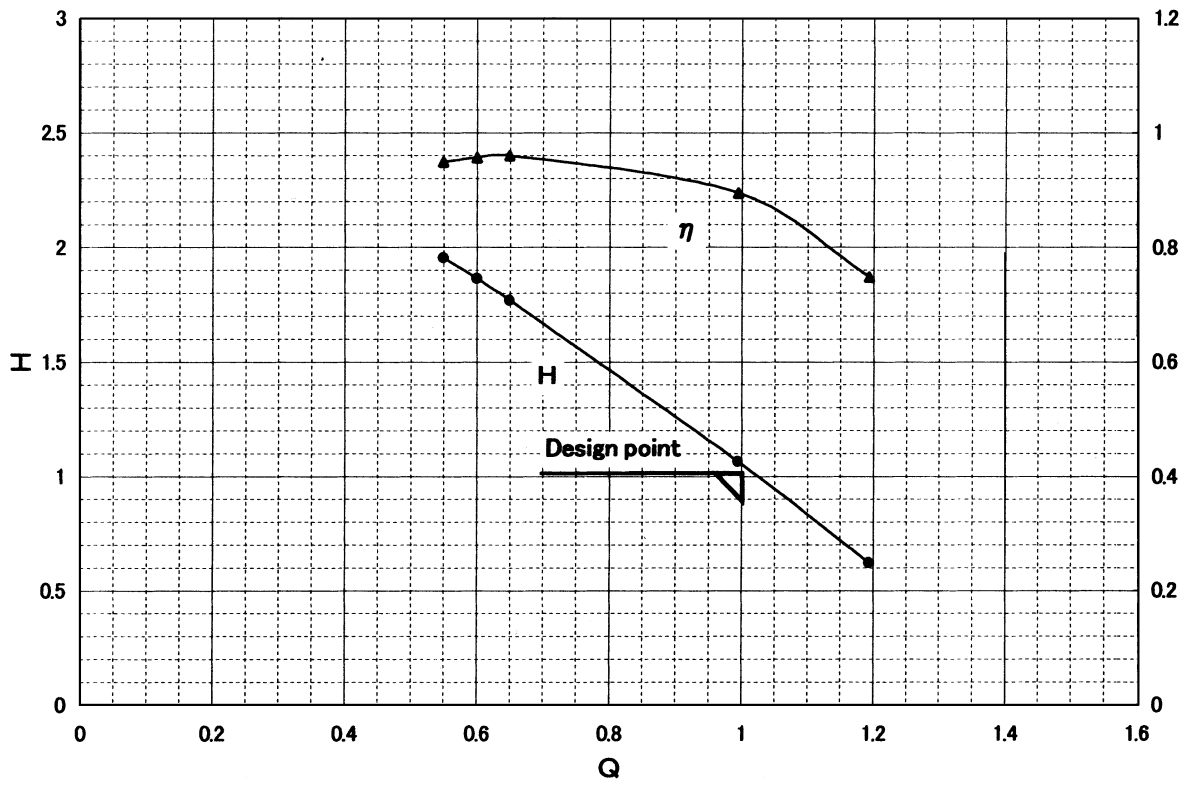

FIGURE 7

Performance curve by analysis of the initial shape. 
8. Results of analysis.

Figure 6 shows the performance curve by analysis of the three-dimensional flow of the optimized impeller shape while the broken line gives the actual measured results. Furthermore, Figure 7 shows the performance curve by analysis of the initial meridional shaped impeller in Figure 2.

Here, flow rate $Q$, head $H$, and efficiency $\eta$ are normalized by each value at the design point of the optimized impeller. A closed flow loop was used to measure these, according to the ISO standard.

The results are as follows:

- The performance curve of the analysis approximates the actual measured results.

- Analysis of the optimized shape indicates efficiency is $8 \%$ greater than that of the initial shape.

\section{CONCLUSIONS}

1. When the coordinates of the impeller inlet hub and shroud are optimized as the design variables, the mixed-flow angle, meridional flow channel section, and the peripheral velocity $U$ change; the distribution of relative velocity $W$ changes quite considerably. Specifically, the shape of the meridional flow channel is greatly changed and the optimization requirement (objective function) is realized.

2. Relative velocity $W$ was set as the objective function since various information is available regarding its relation to pump performance such as efficiency and on its flow mechanism. Even when pressure loss, which directly relates to pump performance, was set as the objective function, the optimization results were almost the same.

3. When analytical results by points of optimization history are observed, the flow mechanisms directly related to performance such as pressure loss cause, etc. are revealed.

4. The time required for the present new design method is short (several hours) and therefore great extendibility may be expected. An attempt was made at optimization with circula- tion distribution as the design parameters in the design flow, shown in Figure 1.

\section{NOMENCLATURE}

$\mathrm{Cu}$ swirl velocity

$H$ head

Hth theoretical pump head

Ns $\quad$ specific speed $\left(\mathrm{m}^{3} / \mathrm{min}, \mathrm{m}, \mathrm{min}^{-1}\right)$

$Q \quad$ flow rate

$\mathrm{RCu}$ circulation

$R \quad$ radius

$U \quad$ peripheral blade speed

$W \quad$ relative velocity

\section{Greek Symbol}

$\eta \quad$ efficiency

\section{Subscripts \\ 1 inlet \\ 2 outlet}

\section{REFERENCES}

Dallenbach, F. 1961. The aerodynamic design and performance of centrifugal and mixed-flow compressors. SAE Technical Progress 3:130 .

Mishina, H. 1978. The study of relative velocity distribution and performance of centrifugal impeller. Trans. JSME 44(384):2689-2698.

Senoo, Y., and Nakase, Y. 1972. An analysis of flow through a mixed flow impeller. Journal of Engineering for Power, Trans. ASME 94(1):43-50.

Vanderplaats, G. N., and Hicks, R. M. 1976. Numerical airfoil optimization using a reduced number of design coordinates. NASA TM $X-73$, p. 151 .

Zangeneh, M., Goto, A., and Takemura, T. 1996. Suppression of secondary flows in a mixed flow pump impeller by application of $3 \mathrm{~d}$ inverse design method: Part 1-Design and Numerical Validation, ASME Journal of Turbomachinery 118:536-543. 

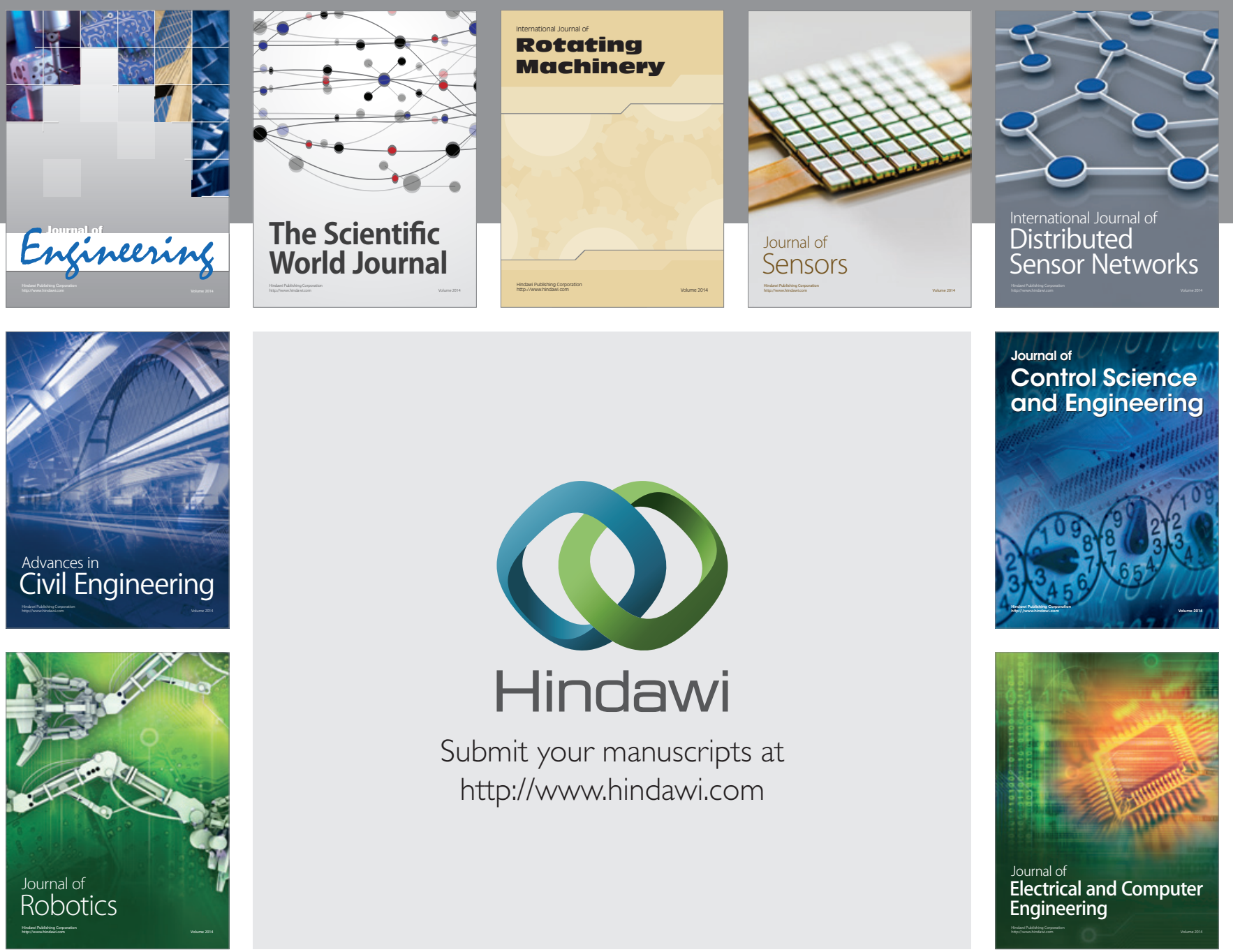

Submit your manuscripts at

http://www.hindawi.com
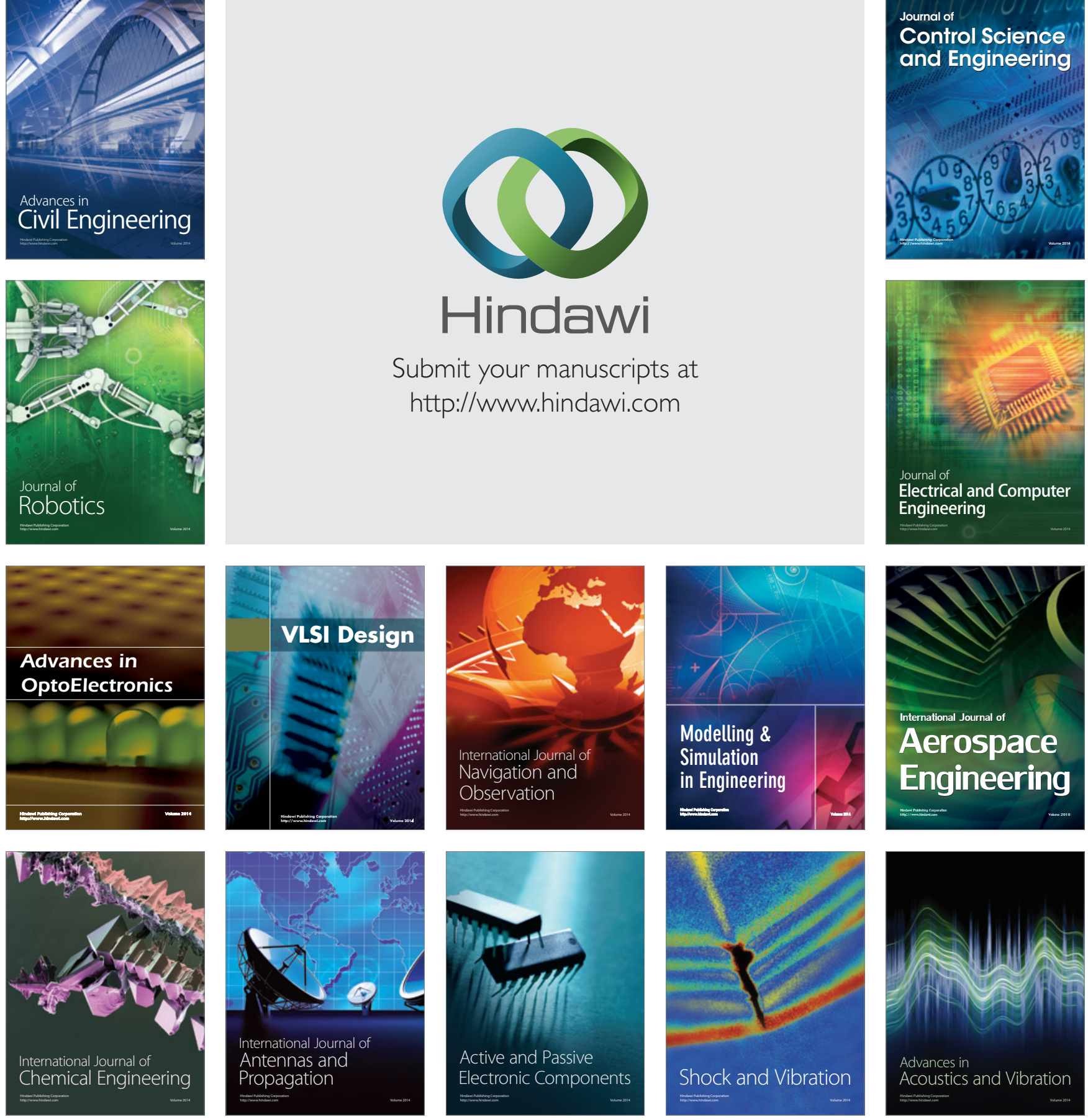